\title{
Split-brain patients neglect left personal space during right-handed gestures
}

\author{
Hedda Lausberg ${ }^{a, b, *}$, Sotaro Kita ${ }^{b}$, Eran Zaidel ${ }^{c}$, Alain Ptito ${ }^{d}$ \\ a Department of Neurology, Free University of Berlin, Hindenburgdamm 30, D-12200 Berlin, Germany \\ ${ }^{\mathrm{b}}$ Max-Planck Institute for Psycholinguistics, Nijmegen, The Netherlands \\ ${ }^{\mathrm{c}}$ Department of Psychology, University of California, Los Angeles, CA, USA \\ ${ }^{\mathrm{d}}$ Cognitive Neuroscience Unit, Montreal Neurological Institute and Hospital, McGill University, Montreal, Canada
}

Received 14 June 2002; received in revised form 22 January 2003; accepted 5 February 2003

\begin{abstract}
Since some patients with right hemisphere damage or with spontaneous callosal disconnection neglect the left half of space, it has been suggested that the left cerebral hemisphere predominantly attends to the right half of space. However, clinical investigations of patients having undergone surgical callosal section have not shown neglect when the hemispheres are tested separately. These observations question the validity of theoretical models that propose a left hemispheric specialisation for attending to the right half of space.

The present study aims to investigate neglect and the use of space by either hand in gestural demonstrations in three split-brain patients as compared to five patients with partial callosotomy and 11 healthy subjects. Subjects were asked to demonstrate with precise gestures and without speaking the content of animated scenes with two moving objects.

The results show that in the absence of primary perceptual or representational neglect, split-brain patients neglect left personal space in right-handed gestural demonstrations. Since this neglect of left personal space cannot be explained by directional or spatial akinesia, it is suggested that it originates at the conceptual level, where the spatial coordinates for right-hand gestures are planned. The present findings are at odds with the position that the separate left hemisphere possesses adequate mechanisms for acting in both halves of space and neglect results from right hemisphere suppression of this potential. Rather, the results provide support for theoretical models that consider the left hemisphere as specialised for processing the right half of space during the execution of descriptive gestures.
\end{abstract}

(C) 2003 Elsevier Science Ltd. All rights reserved.

Keywords: Callosal disconnection; Hemispheric specialisation; Hemi-spatial neglect; Gesture space; Directional akinesia; Partial callosotomy

\section{Introduction}

In light of the clinical evidence showing that unilateral neglect occurs more often and more severely after right hemisphere damage (see review in Bisiach, 1999), several theoretical models of hemi-spatial neglect have assumed the existence of hemispheric asymmetries (Bisiach, 1999; Heilman, Watson, \& Valenstein, 1993; Kinsbourne, 1993; Mesulam, 1981). While these models differ in several aspects, they all agree that the left hemisphere is specialised for attending to the right half of space.

However, split-brain subjects do not show salient persistent neglect in situations involving the independent left hemisphere, such as somato-sensory stimulation (Campbell, Bogen, \& Smith, 1981; Geschwind \& Kaplan, 1962), find-

\footnotetext{
* Corresponding author. Tel.: +49-30-8445-2276; fax: +49-30-8445-4264.

E-mail address: hedda@zedat.fu-berlin.de (H. Lausberg).
}

ing hidden figures in complex drawings (Zaidel, 1979), choosing options in the left space of Ravens' progressive matrices (Zaidel, Zaidel, \& Sperry, 1981), touching and moving the left hemibody, or in a rod bisection experiment with simultaneous performance by the right and left hands (Plourde \& Sperry, 1984). Lateralised tests used with patients with complete callosal disconnection indicate that each hemisphere receives visual information from both visual hemifields and can react appropriately in directing attention (Holtzman, Volpe, \& Gazzaniga, 1984; Lausberg, Göttert, Münßinger, Boegner, \& Marx, 1999). Joynt (1977) has explained that inattention syndromes in split-brain patients are uncommon, " because of the redundancy of the information systems, the disinhibition of the separated hemisphere, the employment of alternative sensory and motor systems, and the persisting consciousness or awareness in both separated hemispheres" (p. 37). This could explain the discrepancy between the absence of clinical neglect in split-brain patients and the presence of neglect in patients 
with right hemisphere damage whose left hemispheres do not develop independent orienting systems. Plourde and Sperry's position (Plourde \& Sperry, 1984) differs from the previous explanation as they emphasise interference from the right hemisphere: "for left hemineglect from right-sided lesions, the leading role of the lesioned right hemisphere may be preserved even though specific spatial and attentional functions are lost, with the result that the left hemisphere is suppressed and unable to exert the intrinsic potential that is present as observed in these tests" (p. 104).

However, in patients with complete callosal disconnection the processing of ipsilateral visual field information is not sufficient to locate the stimulus precisely, guide a manual response to the visual target (Holtzman et al., 1984), and react in a specific manner to figural features of the stimulus, e.g. the direction of an arrow (Lausberg et al., 1999). A hemispheric bias for attending to contralateral environmental space was reported by Làdavas, Del Pesce, Mangum, and Gazzaniga, 1994. In a cued-target detection task, a callosotomy patient showed left hemisphere dominance with a rightward attentional bias (Berlucchi, Aglioti, \& Tassinari, 1997). These findings indicate that under certain experimental conditions, phenomena reminiscent of those seen in patients with neglect from unilateral hemisphere damage are found in split-brain patients.

Furthermore, cases of patients with spontaneous callosal disconnection and left hemineglect in left hemisphere functions (Goldenberg, 1986; Kashiwagi, Kashiwagi, Nishikawa, Tanabe, \& Okuda, 1990; Sine, Soufi, \& Mahendra, 1984) challenge particularly Plourde and Sperry's neglect hypothesis. In these three studies, all patients displayed the classical callosal disconnection syndrome indicating that the interaction between the two hemispheres was severely impaired. If Plourde and Sperry were correct in their assumption, right hemisphere interference via the corpus callosum would have been, at the very least, reduced, and the left hemisphere would have been able to exert its intrinsic potential. However, these patients displayed neglect of left space in left hemisphere functions. The situation is further complicated by the observation of left-sided extinction-like asymmetry during visual search in normal subjects, which was reversed in a complete commissurotomy patient (Pollmann \& Zaidel, 1998). These data are consistent with right hemisphere dominance for bilateral visual orienting, augmented by a receptor field-based attentional gradient from high-right to low-left.

In order to help clarify these apparently conflicting positions, the present study addresses the question of neglect in split-brain patients by testing the spontaneous use of space in communicative gestures. McNeill and Pedelty (1995) observed in the split-brain patient NG that her iconic right-hand gestures were exclusively displayed in right personal space. For the same patient, Plourde and Sperry (1984) reported no neglect in clinical tests. There are several pieces of evidence that neglect is often a task-dependent phenomenon that can become manifest in certain conditions but not in others. The stimulus per se (Halligan \& Marshall, 1988; Tegnér \&
Levander, 1991), simultaneous hand movements (Robertson \& North, 1992, 1994), simultaneous optokinetic stimulation (Bisiach, Pizzamiglio, Nico, \& Antonucci, 1996), experimental test condition as compared to routine clinical tasks (Berlucchi et al., 1997; Làdavas et al., 1994), and behavioural goal (Karnath \& Niemeier, 2002) all can influence the manifestation of neglect signs. Heilman et al. (1993) emphasised the necessity to investigate both 'exogenously evoked' and 'endogenously evoked' movements in the analysis of action-intentional disorders. Along similar lines, the investigation of the spontaneous use of space in gestures might reveal signs of neglect that are not observed in clinical tests. The manifestation of spontaneous preference for right hemi-space by the right hand might be overridden if a specific use of space in motor action is required. Furthermore, the use of space determined by imagined targets in space such as in the present experiment might differ from the use of space specified by actual visual targets such as in Raven's matrices (Zaidel et al., 1981) or the ITPA visual closure subtest (Zaidel, 1979), or specified by body parts (Plourde \& Sperry, 1984).

In the present study, subjects were asked to precisely demonstrate with gestures the content of animations of two geometric objects moving on a horizontal line in a scene defined by left and right borders and divided by a centre into left and right hemi-spaces. In a pilot study with the same design, healthy subjects demonstrated with gestures the scene by placing its centre in front of their body-midlines. The right border of the scene was represented in their right personal spaces, and the left border in their left personal spaces. Following McNeill and Pedelty's observation (McNeill \& Pedelty, 1995), we hypothesised that the split-brain patients would neglect left personal space in their gestures by the right hand. To test this hypothesis, the gestures of each hand were evaluated by coding the spontaneous placement of the centre, as well as the left and right borders in relation to the body-midline.

The basic assumption of the present study is that in split-brain patients, the right-hand gestural performance reflects the use of space mediated by the left hemisphere while left-hand performance reflects the opposite, i.e. space use by the right hemisphere. Consideration should, however, be given to the fact that over time, callosotomy patients develop varying degrees of ipsilateral motor control of the limbs (Gazzaniga, Bogen, \& Sperry, 1967; Trope, Fishman, Gur, Sussman, \& Gur, 1987; Volpe, 1985; Volpe, Sidtis, Holtzman, Wilson, \& Gazzaniga, 1982; Zaidel \& Sperry, 1977). However, ipsilateral control has, thus far, been documented in experiments with sensory input lateralised to a single hemisphere with, in addition, the explicit command to respond with the ipsilateral limb. In an experiment with tachistoscopic presentation of hand postures, NG and LB, two patients with complete commissurotomy, were able to imitate on command with their ipsilateral left hand 80-90\% of the tasks while with their ipsilateral right hand, they could only do so on $25 \%$ of the tasks (Zaidel \& Sperry, 1977). 
In the same session, they were submitted to non-lateralised tasks such as performing meaningful movements to verbal commands. A left-hand dyspraxia was then documented, suggesting that ipsilateral control was not sufficiently used in the absence of lateralised input. In the present study, the animations were presented in a non-lateralised manner and, in addition, no command was given concerning which hand to use. The subject's use of the right or left hand was therefore completely spontaneous. We are not aware of any reports about split-brain patients making use of ipsilateral control in spontaneous motor actions. The only reports that deal with spontaneous motor behaviour in split-brain patients describe disorders such as the autonomous hand syndrome (Zaidel, Iacoboni, Zaidel, \& Bogen, in press), which has also been described as the "alien hand syndrome" (e.g. Feinberg, Schindler, Flanagan, \& Haber, 1992), as well as conditions pertaining to intermanual conflict (Zaidel et al., in press), also termed diagonistic dyspraxia (Akelaitis, 1945). If occurring in patients with callosal disconnection, these phenomena imply that the right hemisphere spontaneously controls the left hand via contralateral pathways (Bogen, 2000; Geschwind et al., 1995; Tanaka, Yoshida, Kawahata, Hashimoto, \& Obayashi, 1996). Therefore, it seems plausible that if the stimulus is presented to both hemispheres and both hands are free to act, the more effective contralateral motor pathway will preferentially be used to perform hand gestures. On these grounds, we investigate the use of space by the right and left hands as representing hemispheric specialisation in the use of space. The assumption of contralateral control is especially well-supported for the right hand of split-brain patients.

\section{Methods}

\subsection{Subjects}

We tested three patients with complete commissurotomy and callosotomy, respectively (AA, NG and GC, respec- tively). As control, we investigated five patients with partial callosotomy (LM, SR, GS, LD, CE) and 11 healthy subjects. The patients' case histories are summarised in Table 1 (for AA and NG, further details of the case histories can be found in Bogen (1969), Milner and Taylor (1972), Zaidel (1998)).

In AA, NG and GC, MRI examination confirmed the extent of the callosotomy, but the status of the anterior commissures could not be evaluated. However, for AA and NG, Bogen (1993) did report severing the anterior commissure (see Bogen, Schultz, and Vogel (1988), for further description of AA and NG). In LM, the section was complete, except for $1 \mathrm{~cm}$ of the mid-trunk as well as fibres in the rostrum of the corpus callosum. GS had a section of 7/8 of the corpus callosum sparing the rostrum and splenium. SR, LD and $\mathrm{CE}$ had a section of the anterior two thirds of the corpus callosum. SR had an additional left fronto-temporal resection and an amygdalo-hippocampectomy. The healthy controls were chosen from a cohort of 122 healthy adults tested neuropsychologically for a previous normative study at the Montreal Neurological Institute and Hospital in 1993/1994.

The three groups were matched as closely as possible according to gender $\left(\chi^{2}=0.154\right.$; exact $\left.P=1.0\right)$ and age ( $F=1.503 ; P=0.252)$. Patient $C E$ had an average IQ rating while the other patients had IQ ratings within the low average range (Wechsler adult intelligence scale-revised). The IQ ratings of the healthy controls were distributed almost evenly within the average range. Handedness was established with a questionnaire currently used at the Montreal Neurological Institute and Hospital. All subjects were right-handed except for one healthy control who was ambidextrous with a strong tendency towards right-handedness.

Sensori-motor testing included passive movement of the fingers, simultaneous stimulation of the hands, pinch or grip strength, free and sequential tapping, and manual dexterity (Grooved or Purdue pegboard). A deficit in passive movement of the fingers was found in AA bilaterally, especially for the right hand, and in NG for the right hand only. For AA, this deficit had been described before and attributed to his left hemisphere lesion (Taylor, 1970). Both patients also had

Table 1

Summary of patients' case histories

\begin{tabular}{|c|c|c|c|c|c|c|}
\hline Patient & Sex & Surgery type & Presence of extracallosal damage & $\begin{array}{l}\text { Age(s) at } \\
\text { surgery(ies) }\end{array}$ & $\begin{array}{l}\text { Age at } \\
\text { testing }\end{array}$ & $\begin{array}{l}\text { WAIS IQ } \\
\text { at testing }\end{array}$ \\
\hline AA & Male & Single stage complete commissurotomy & Left fronto-parietal, right frontal & 14 & 50 & 79 \\
\hline GC & Male & Three stage complete callosotomy & $\begin{array}{l}\text { Left auditory cortex, left optic } \\
\text { radiation/visual cortex (based on } \\
\text { functional defects) }\end{array}$ & $33,35,38$ & 46 & 78 \\
\hline NG & Female & Single stage complete commissurotomy & Left posterior temporal, right central & 30 & 66 & 81 \\
\hline LM & Male & $\begin{array}{l}\text { Two-stage callosotomy, ca. } 1 \mathrm{~cm} \text { fibres } \\
\text { in mid-truncus and rostrum spared }\end{array}$ & $\begin{array}{l}\text { Left temporal atrophy, bi-occipital } \\
\text { lesions left }>\text { right }\end{array}$ & 12,13 & 23 & 76 \\
\hline GS & Male & $\begin{array}{l}\text { Single stage callosotomy }(7 / 8), \\
\text { rostrum and splenium spared }\end{array}$ & Bilateral parieto-occipital & 24 & 29 & 71 \\
\hline SR & Male & Two-stage anterior callosotomy & $\begin{array}{l}\text { Left fronto-temporal resection, left } \\
\text { amygdalohippocampectomy }\end{array}$ & 24,25 & 37 & 84 \\
\hline LD & Female & Single stage anterior callosotomy & Resection of craniopharyngioma & 40 & 59 & 88 \\
\hline $\mathrm{CE}$ & Female & Single stage anterior callosotomy & - & 36 & 45 & 92 \\
\hline
\end{tabular}


astereognosis involving the right hand selectively (Zaidel, 1998). In the partial callosotomy group, LM showed a bilateral deficit in perceiving light touch and position sense. Sensory extinction for the left hand occurred only in two partial callosotomy patients (LD, GS) and in one healthy control. No extinction errors were found in the patients with complete callosotomy.

There was no evidence of paresis in any participant as determined by pinch and grip strength examination. The pegboard and simple tapping tests showed some general slowing in all patients, a result possibly associated with their anti-epileptic medication. Bimanual synchronous, symmetrical coordination (Purdue pegboard) was adequate in the patients with complete callosotomy. These results concur with previous examinations in split-brain patients by Campbell et al. (1981) including AA and NG. Bimanual synchronous, asymmetrical coordination (sequential tapping) was tested only in the partial callosotomy patients and the healthy controls and results were in the normal range. Overall, the essentially normal motor tests results concur with those obtained from a similar test battery in a previous investigation on partial and complete callosotomy patients (Zaidel \& Sperry, 1977).

In addition to sensori-motor test-batteries, several classical tests of interhemispheric interaction were carried out. Agraphia was tested by the dictation of 10 words, first with the left hand, then with the right. The 38 samples with the written words (19 subjects $\times 2$ hands) were presented in a random order to two independent blind raters who coded the written words according to the criteria used in a previous experiment on callosal agraphia (Lausberg et al., 1999). The inter-rater agreement was high (Pearson correlation $r=$ 0.98 ) and the healthy control group was used to define the normal range of performance. With the left hand, the three split-brain patients AA, GC and NG, and the partial callosotomy patient $\mathrm{LM}$, performed below the normal range. With the right hand, the performances of AA and LM were also subnormal but much better than with the left hand.

Visuo-constructive abilities were assessed with the copy of the Taylor complex geometric figure as well as with the simple geometric drawings of the Wechsler memory scale (WMS) cards A and C. First the right hand was used, then the left. The subjects' right- and left-hand copies were presented in random order to independent blind raters. For the Taylor figure, inter-rater agreement of three raters was high (raters 1 and 2: $r=0.99$ (Pearson correlation), raters 1 and 3: $r=0.90$ and raters 2 and 3: $r=0.90$ ); for the WMS drawings, the agreement of two raters was $r=0.84$. With the Taylor figure, AA, GC, NG and the partial callosotomy patient GS performed below the normal range with either hand when compared to the healthy control group. Left-hand performances were only slightly better than right-hand performances. On the WMS drawings, GC and NG performed below the normal range with either hand; the partial callosotomy patient LM obtained scores slightly below the normal range with the left hand. In addition, no evidence of object-centred neglect was found in any subject with the copies of the Taylor figure, the WMS drawings, or 10 other drawings (square, triangle, rectangle, star, house, car, three-dimensional square, pyramid, three-dimensional rectangle, cup).

Left-hand dyspraxia with conceptual errors in pantomime to visual presentation of objects was observed in the three split-brain patients and to a very minor degree in the partial callosotomy patient GS (Lausberg, Cruz, Kita, Zaidel, \& Ptito, 2003).

To test sensori-motor transfer, the blindfolded subject had to raise the finger of one hand corresponding to the finger that was touched on the other hand. While in the healthy control group the mean percentage of errors was 3\% (range 0-12\%), it was $74 \%$ (range $72-75 \%$ ) in the split-brain group. In the partial callosotomy group, the mean percentage of errors was $13 \%$ (range 1-23\%) with LM, SR and GS performing below the range of the healthy control group. No salient group differences occurred in the two control experiments in which (a) the blindfolded subject had to raise the same finger that was touched; and (b) the subject had to raise in free view the finger of one hand corresponding to the finger that was touched on the other hand.

To summarise, the three patients with complete callosotomy showed a classical disconnection syndrome with left-hand agraphia, left-hand dyspraxia, and a lack of sensori-motor transfer. The fact that the split-brain patients had a significant visuo-constructive deficit not only in the right hand-as classically described for callosal syndromes - but in both hands, concurs with a previous investigation of AA and NG (Bogen, 1969). In the partial callosotomy group, differences between performances in tests of interhemispheric interaction were related to the extent of corpus callosum section. In LM and GS, the callosotomy includes posterior parts of the corpus callosum; in $\mathrm{SR}, \mathrm{LD}$ and CE, the section is limited to the anterior corpus callosum. LM had a left-hand agraphia, a visuo-constructive deficit in both hands, and an impaired sensori-motor transfer. GS showed a minor degree of left-hand dyspraxia as well as a visuo-constructive deficit in both hands and a sensori-motor transfer impairment. In the three patients with anterior callosotomy, only SR performed in one test below the normal range. Therefore, in the evaluation of the space use in gestural demonstrations of the partial callosotomy group, special attention was paid to LM and GS as they differed from the other patients whose sections were restricted to the anterior part of the corpus callosum.

\subsection{Materials and procedures}

Subjects were presented 24 animations with an average duration of $30 \mathrm{~s}$. In the animations, two geometric objects move on a horizontal line in a scene.

The scene was defined by a centre and by left and right borders. These three landmarks were functionally defined as they represented the locations at which the objects appeared 
or disappeared from the scene, or the point at which two objects met. The 24 animations shared the following temporal structure: phase 1, each animation started with an object located at the centre of a scene; phase 2, the second object appeared on the scene at either the right ( $n=12$ animations) or the left borders ( $n=12$ animations), and moved towards the object at the centre; phase 3, the second object either stopped close to the centred object ( $n=16$ animations) or touched it $(n=8)$; phase 4 , (a) both objects remained at the centre $(n=6)$; (b) the second object moved back to the starting point $(n=6)$; (c) the first object moved to the other border, i.e. in the direction opposite that of the second object $(n=8)$; or (d) both objects continued to move towards the other border $(n=4)$.

In addition to the motion path of an object, object motion type and object shape varied. The objects could either simply be in motion or, they could move in a rolling or jumping manner. There were three variations of object shape and colour, a red ball, a blue square, and a green triangle. These variations were introduced to maintain the subjects' attention during the 24 trials.

The animations were presented on a TV screen that was placed $2.5 \mathrm{~m}$ in front of the subject. On the screen, the height of the objects was $5 \mathrm{~cm}$. The subjects had to demonstrate with gestures the content of each animation without speaking and were asked to start with their demonstration immediately after the animation had been shown on the TV screen. The following instruction was given to the subjects: "Please demonstrate in gesture, without speaking and as precisely as possible, what happened in the animation. Your gestural demonstration should be so precise that an observer who does not know the animation can narrate the content of the animation by watching your hands". For the first five trials, the investigator provided feedback by verbally reporting on-line what she comprehended from the subjects' gestural demonstrations. In the feedback, the terms 'left' and 'right' were adapted to the subject's perspective, e.g. "I see ... a ball ... rolling from the [subject's] left ... to the right". The subjects became aware of the discrepancies between what they intended to express and what was recognisable for the observer and, thereby, learned to be very explicit in their demonstrations. No specific instructions were given to the subjects such as to depict a certain object shape or to show a contact between the two objects.

Each subject's gestures were taped with a video camera (Sony DCR TRV900E miniDV camcorder) placed close to the TV screen. The videotapes were digitised to MPEG 1 format. This procedure permitted use of the movement analysis program Media Tagger (Brugman \& Kita, 1995) for the evaluation of the movie files. With this software, a segment of a movie can be selected and tagged with a value. In the present study, the complete gestural response to one animation was a coding unit. An independent trained rater, blind to the subjects' diagnoses and to the hypotheses of the study, evaluated the gestures with a coding system consisting of one ordinal and six nominal variables: (a) detail score; (b) hand use; (c) centre placement in the horizontal dimension; (d) centre placement in the sagittal dimension; (e) border placement in the horizontal dimension; (f) wrong object touch display; and (g) trunk orientation. The first author also rated the gestures in order to verify inter-rater agreement.

\subsubsection{Detail score}

A detail score was coded that reflected the accuracy of the gestural demonstration: (a) information about the manner of the object movement(s), i.e. simple movement; moving in a rolling manner; moving in jumping manner; (b) the path(s), i.e. moving to one direction only or moving back and forth; (c) the shapes of the two objects, i.e. ball, square, triangle; and (d) contact between the two objects, i.e. touch, no touch (distant contact). The scoring was 0 points for an incorrect, incomplete, or missing detail; and 1 point for a correct detail. The maximum detail score sum was 162 . In addition, details concerning the movement paths of the objects were grouped according to whether they occurred in the right hemi-space of the animation (maximum 24 points) or in the left hemi-space (maximum 24 points) (within the right and left hemi-spaces, there were equal numbers of object movements to the right and to the left). The inter-rater agreement for the detail score was $r=0.86$ (Pearson correlation).

\subsubsection{Hand use}

To demonstrate the content of the animation scene without speaking, the subjects were free to choose their right and/or left hands to represent the objects, their spatial positions, their motion paths, their manners of movement, and their shapes. For each gestural response, the spontaneous use of the hand was coded separately as follows: (1) the hand was not used in the response; (2) the hand by itself represented an object or an object feature, i.e. independently from the other hand; (3) the hand was used only as part of a two-handed object representation, i.e. the two hands jointly represented an object, e.g. shaped a ball. For each response and hand, the three categories were mutually exclusive. The inter-rater agreement (Cohen's kappa) for right hand use was $r=0.83$ and $r=0.88$ for left hand use.

In addition, for each response requiring independent use of at least one hand, the hand that started the response was noted.

\subsubsection{Centre placement in the horizontal dimension}

In the animations, the centred object marked the centre of the scene where the two objects also met (Fig. 1). In a subject's gestural demonstrations, the centre of the scene could be placed either in front of the body-midline, or the centre could be shifted towards the right or left. Each centre placement was coded separately for the right and left hands, with the following mutually exclusive categories: (1) the hand places the centre of the gestured scene in front of the body-midline (no centre shift); (2) the hand shifts the centre to the side contralateral to the hand, i.e. the right hand shifts the centre to the left; (3) the hand shifts the centre to the 


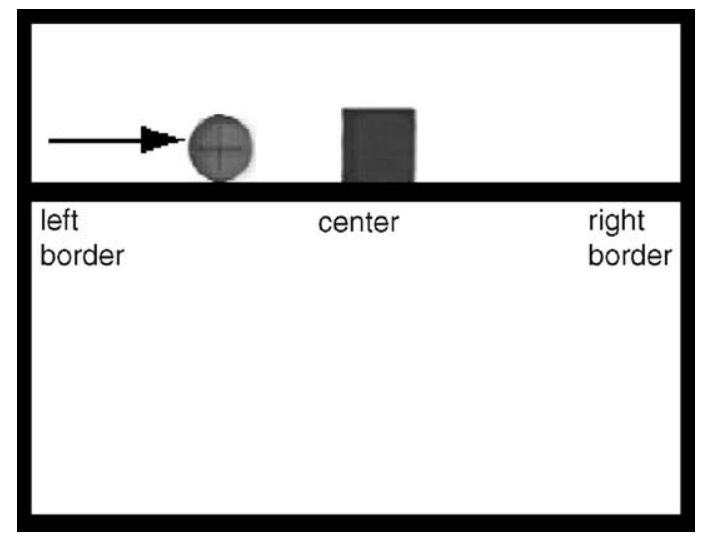

Fig. 1. An example of an animation: a square is located in the centre of the scene. A ball moves from the left border to the centred object (in the figure, the motion direction is indicated by the arrow). The words 'left border', 'centre', and 'right border' and the arrow are not part of the original animations.

side ipsilateral to the hand, i.e. the right hand shifts the centre to the right. The inter-rater agreement (Cohen's kappa coefficient) for centre placement was $r=0.77$.

\subsubsection{Centre placement in the sagittal dimension}

In the present study, the movement analysis was based on video recordings facing the subject. With this method the estimation of a hand position in the sagittal dimension (depth) was not reliable. Therefore, for the evaluation of the centre placements in the sagittal dimension, the positions of both hands were compared with each other. The following mutually exclusive categories were used: (1) the right hand places the centre closer to the body than the left hand; (2) the left hand places the centre closer to the body than the right hand; (3) the two hands place the centre at the same level in the sagittal dimension. The inter-rater agreement (Cohen's kappa coefficient) for sagittal centre placement was $r=1.00$.

\subsubsection{Border placement in the horizontal dimension}

The placements of the left and right borders of the gestured scene were evaluated with reference to the body-midline and the right and left personal spaces. Each border placement was coded separately for the right and left hands with the following mutually exclusive categories: (1) the right border was placed in the subject's right personal space (corresponding border placement); (2) the right border was shifted to the left and placed in front of the subject's midline or in the left personal space (non-corresponding border placement); (3) the left border was placed in the subject's left personal space (corresponding border placement); (4) the left border was shifted to the right and placed in front of the subject's midline or in the right personal space (non-corresponding border placement). Border shifts towards the ipsilateral side of the performing hand, i.e. the right border shifted to the right, were theoretically possible but would not be coded because of individual variations in the peripheral extension of the gestured scene. The inter-rater agreement for border placement (Cohen's kappa) was $r=0.77$.

\subsubsection{Wrong object touch display}

In eight of the 24 animations, the second object approached the centred object and touched it. For those eight animations, a wrong contact display was noted, i.e. the hand that represents the stationary centred/to-be-touched object approaches and actively touches the hand that should have been making the movement. The inter-rater agreement (Cohen's kappa) was $r=0.89$.

\subsubsection{Trunk rotation}

Trunk rotation around the vertical axis of the body was noted if it occurred in a gestural response. The inter-rater agreement for this variable was $r=0.71$ (Cohen's kappa).

\section{Results}

Due to the small sample sizes and related problems with assumptions, we choose to treat the following analyses as exploratory in nature, and we interpret the results with appropriate caution as trends that should be investigated with larger samples.

\subsection{Detail score}

Table 2 shows for each subject the detail scores and the subscores for details in each hemi-space and the respective group means.

In the detail score, the split-brain patients were below the normal range compared to the healthy group and below the range of the partial callosotomy patients. In all three groups, there was no salient difference between the score for details in the left hemi-space and the score for details in the right hemi-space of the animation.

\subsection{Hand use}

In the 24 responses in the experiment, the mean number of responses in which the right hand was used independently was 21.95; the mean number of responses in which the right hand was only used as part of a two-handed representation together with the left hand was 1.84; and the mean number of responses in which the right hand was not used was 0.21 . The mean number of responses in which the left hand was used independently was 16.95; only used as part of a two-handed representation together with the right hand 3.53; and not used 3.53. The subjects' numbers of responses in each category were submitted to a mixed ANOVA with one between-subjects factor (group) and two repeated factors (hand, use) with two levels each (right hand versus left hand; independent versus two-handed). The effects of hand $(F=5.98 ;$ d.f. $=1 ; P=0.026)$, use $(F=48.86$; d.f. $=1$; 
Table 2

Detail scores and the subscores for details in each hemi-space for each subject and respective group means

\begin{tabular}{ccll}
\hline & Detail score & Left space score & Right space score \\
\hline \multicolumn{2}{l}{ Complete callosotomy } & & \\
AA & 88 & 19 & 21 \\
GC & 74 & 19 & 18 \\
NG & 86 & 23 & 22 \\
Mean & 82.7 & 20.3 & 20.3 \\
Partial callosotomy & & \\
LM & 105 & 23 & 21 \\
GS & 101 & 23 & 23 \\
SR & 126 & 24 & 23 \\
LD & 127 & 24 & 23 \\
CE & 100 & 22 & 23 \\
Mean & 111.8 & 23.2 & 22.6 \\
Healthy controls & & \\
1 & 118 & 24 & 23 \\
2 & 129 & 24 & 23 \\
3 & 122 & 24 & 24 \\
4 & 128 & 24 & 22 \\
5 & 132 & 24 & 23 \\
6 & 121 & 23 & 24 \\
7 & 104 & 24 & 23 \\
8 & 128 & 24 & 24 \\
9 & 125 & 24 & 22 \\
10 & 130 & 24 & \\
11 & 126 & 24 & \\
Mean & 123.9 & 23.9 & \\
\hline
\end{tabular}

$P=0.000)$, and the interaction of hand $\times$ use $(F=13.63$; d.f. $=1 ; P=0.002$ ) were significant. There were no effects of group and of any interaction with group. Hence, the split-brain patients did not differ in their patterns of hand use from the two control groups. As the comparison of the gestures by the independent use of the right and left hands was relevant in the present study, for each hand, responses in which that hand was only involved in two-handed demonstrations and responses in which that hand was not used were excluded from further evaluation. As the right hand was used more often independently for gestures than the left hand, the statistical analyses in the following evaluations are based on proportions, i.e. the number of left hand responses in a certain category is divided by the total number of responses with independent left hand use and analogously for the right hand.

In addition, for each subject, the proportion of responses with the left hand starting (number of responses with left hand starting/number of responses with one or both hands independently used) and of responses with the right hand starting were calculated. The proportions of left and right hand starts for each subject were submitted to a mixed ANOVA with one between-subjects factor (group) and one repeated factor (hand start) with two levels (right hand start versus left hand start). While there was a significant right hand preference for starting the response $(F=6.137$; d.f. $=1$; $P=0.027)$, no significant differences between the groups (interaction of group $\times$ hand start) were found.

\subsection{Centre placement in the horizontal dimension}

Fig. 2 shows, separately for the right and left hands, the group means and standard deviations of the numbers of centre placements with the centre placed in front of the body-midline, with the centre shifted to the ipsilateral side of the gesturing hand, and with the centre shifted to the contralateral side of the gesturing hand.

Examination of Fig. 2 shows that with either hand the two control groups (partial callosotomy and healthy controls) placed the centre of the gestured scene in front of their body-midlines (white columns). In the patient group

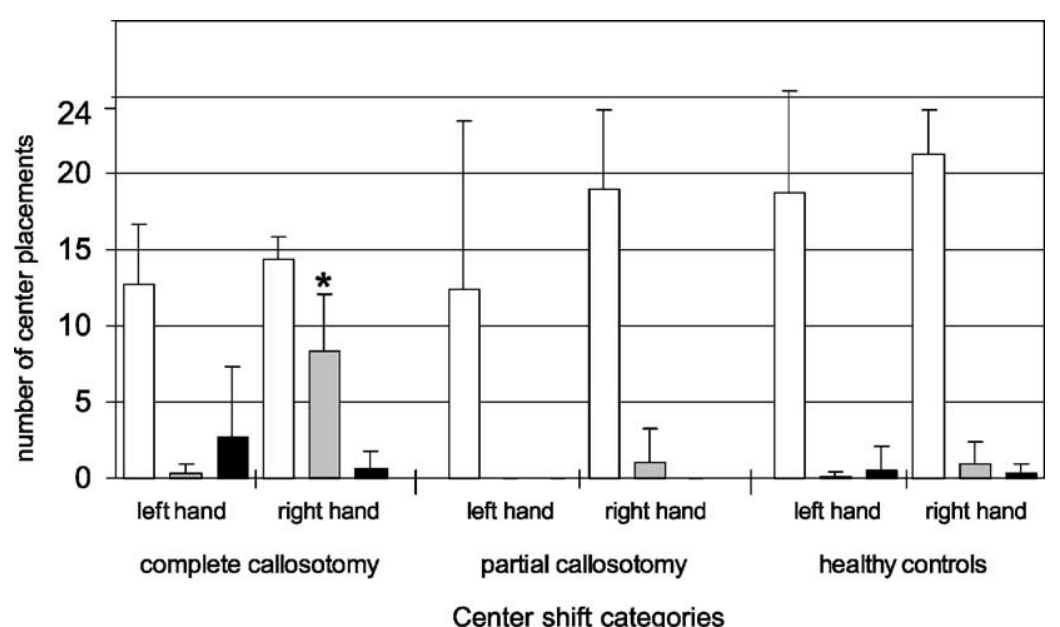

Fig. 2. Placing of the centre of the scene in relation to the body-midline. Group means and standard deviations of the numbers of centre placements in which the centre is placed in front of the body-midline (white columns $(\square)$ ), the centre is shifted to the side ipsilateral of the performing hand (grey columns $(\square)$ ), and the centre is shifted to the side contralateral of the performing hand (black columns $(\square)$ ). Centre placements by the right and left hands are shown separately. Asterisk $\left(^{*}\right)$ stands for significant interaction $(P=0.035)$. 


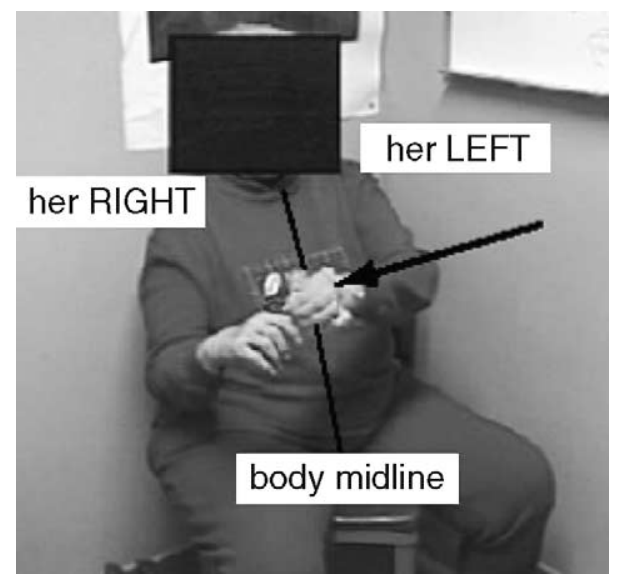

Fig. 3. Patient NG demonstrates the animation shown in Fig. 1. Her right hand represents the centred square and her left hand represents the ball. The centre of the gesturally demonstrated scene is defined by the position of her right hand (centred square) and is shifted to the right in relation to her body-midline. Her left hand (ball) crosses the body-midline to approach the right hand (square) and thereby, also shifts the centre to the right.

with partial callosotomy, all right hand centre shifts to the right (grey column) were conducted by a single patient (GS). In contrast, the split-brain patients frequently shifted the centre of the scene to the ipsilateral right side in their right-handed demonstrations (right hand: grey column with asterisk). The centre shifts by the left hand to the contralateral right side (left hand: black column) were performed by a single split-brain patient (NG) and occurred only if her right hand had already placed the centre with a rightward shift (Fig. 3).

The subjects' proportions of ipsilateral and contralateral centre shifts in all centre placements were calculated for each hand, i.e. the number of right hand centre shifts to the ipsilateral right side was divided by the total number of right hand centre placements (number of ipsilateral shifts/[number of midline placements + number of ipsilateral shifts + number of contralateral shifts]). The proportions of the ipsilateral and contralateral centre shifts were submitted to a mixed ANOVA with one between-subjects factor (group) and two within-subjects factors (hand, direction of shift) with two levels each (right hand versus left hand; shift to side ipsilateral to performing hand versus shift to side contralateral to performing hand). There were significant effects of group $(F=9.18$; d.f. $=2 ; P=0.002)$, hand $(F=18.57$; d.f. $=1 ; P=0.001)$, hand $\times$ group $(F=7.38 ;$ d.f. $=2 ; P=0.005)$, direction of $\operatorname{shift}(F=$ 16.74; d.f. $=1 ; P=0.001)$, direction of shift $\times$ group $(F=7.89 ;$ d.f. $=2 ; P=0.004)$, hand $\times$ direction of shift $(F=9.73$; d.f. $=1 ; P=0.007)$ and hand $\times$ direction of shift $\times$ group $(F=4.37$; d.f. $=2 ; P=0.035)$ (see asterisk in Fig. 2). The split-brain patients differed significantly from the two control groups as they performed more centre shifts with the right hand to the right side.

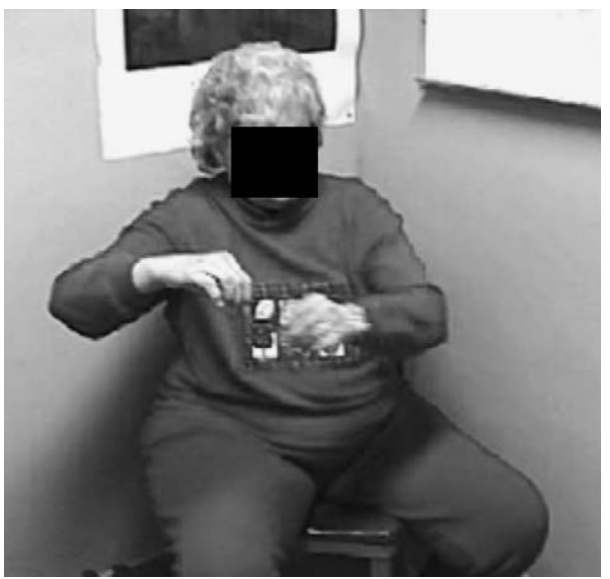

Fig. 4. Patient NG demonstrates an animation in which the left hand object meets the right hand object at the centre (similar to the animation shown in Fig. 1). In addition to a rightward shift of the centre in both hands (compare Fig. 3), the left hand places the centre further away from the body than the right hand.

\subsection{Centre placement in the sagittal dimension}

Only the split-brain patient NG displayed discrepancies between the centre placements by the right and left hands in the sagittal dimension. In four responses, the left hand placed the centre at the same level in the horizontal dimension but further away from the body than the right hand. The sagittal discrepancy always co-occurred with left hand centre shifts to the right. Fig. 4 shows that in addition to a rightward shift of the centre in both hands, the left hand places the centre further away from the body than the right hand.

\subsection{Border placement}

Fig. 5 shows the group means and standard deviations of the numbers of corresponding and non-corresponding left and right border placements, separately for each hand (note that a left border shift was coded if the left border was placed in front of the body-midline or further in the right gesture space and vice versa for the right border shifts).

With either hand, the two control groups represented the right borders in their right personal spaces and the left borders in their left personal spaces (corresponding border placement: white columns). In the patient group with partial callosotomy, all left border shifts to the right by the right hand (right hand/left b.: grey column) occurred in a single patient (GS). In their right-handed demonstrations, the split-brain patients frequently shifted the left borders towards the right and placed them in front of their body-midlines (right hand/left b.: grey column with asterisk). With their left hands, the split-brain patients showed performances comparable to those of the two control groups (left hand: white columns).

For each hand, the proportions of shifts of the border ipsilateral to the performing hand and of shifts of the border 


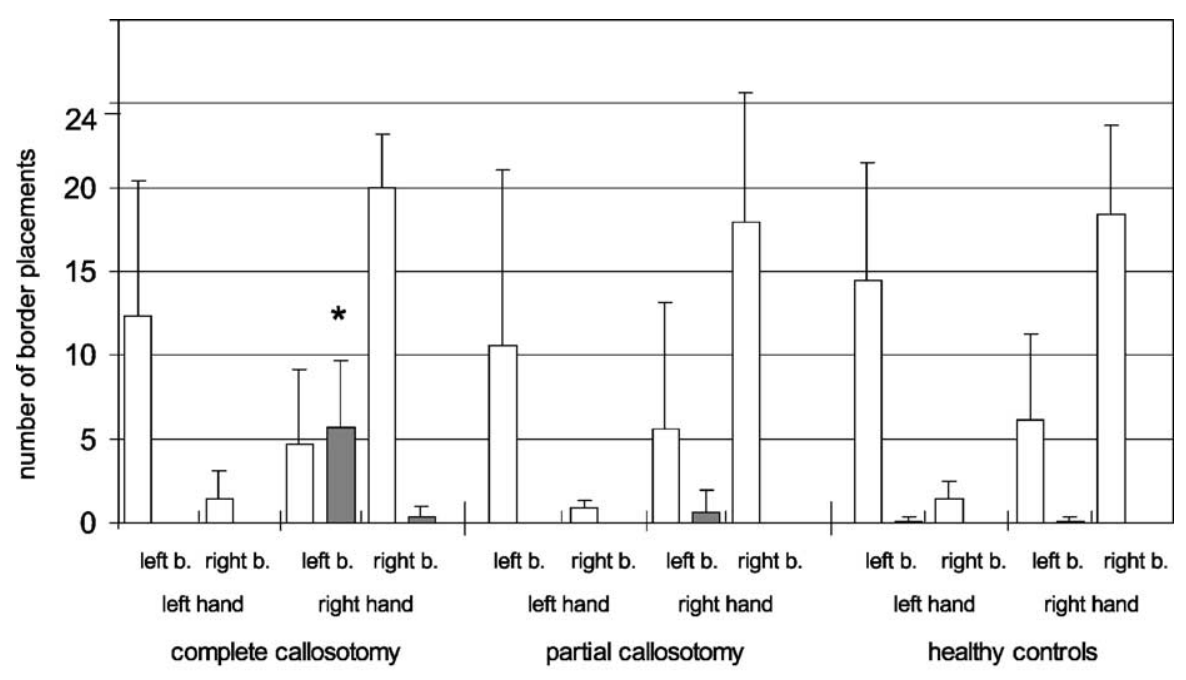

Border placement categories

Fig. 5. Placements of the left borders (left b.) and the right borders (right b.) of the scene. The data show group means and standard deviations of the numbers of border placements in the corresponding personal spaces (white columns $(\square)$ ) and of non-corresponding border placements (grey columns $(\square)$. Asterisk $(*)$ stands for significant interaction $(P=0.000)$.

contralateral to the performing hand were calculated, i.e. for the right hand, the number of ipsilateral right border shifts were divided by the total number of right and left border placements by the right hand (number of right border shifts/[number of right border corresponding placements + number of left border corresponding placements + number of right border shifts + number of left border shifts]). The subjects' proportions were submitted to a mixed ANOVA with one between-subjects factor (group) and two within-subjects factors (hand, shifted border) with two levels each (right versus left hand; border ipsilateral versus border contralateral to performing hand). There were significant effects of group $(F=22.65$; d.f. $=2$; $P=0.000)$, hand $(F=46.28 ;$ d.f. $=1 ; P=0.000)$; hand $\times$ group $(F=40.0$; d.f. $=2 ; P=0.000)$, shifted border $(F=33.25 ;$ d.f. $=1 ; P=0.000)$, shifted border $\times$ group $(F=21.72$; d.f. $=2 ; P=0.000)$, hand $\times$ shifted border $(F=28.95$; d.f. $=1 ; P=0.000)$ and hand $\times$ shifted border $\times$ group $(F=16.01 ;$ d.f. $=2$; $P=0.000$ ). The split-brain patients differed significantly from the two control groups as their right hands shifted the left border to the right and placed it in front of their bodymidlines.

\subsection{Wrong object touch display}

Wrong touches, i.e. touching and to-be touched hands are mixed-up, were only performed by the patient NG. In four of four responses in which the object represented by the right hand should have touched the object represented by the left hand she instead displayed touches with the left hand actively approaching and touching the right hand, despite the fact that the animations required the opposite pattern. With her right hand, NG never actively approached and touched the left hand.

\subsection{Trunk rotation}

Trunk rotation was found only in NG. In eight gestural demonstrations, NG rotated her trunk around the body axis to the left. In six of these eight responses with trunk rotation, her right hand represented an object that moved in the animation from the centre to the left border. Instead of moving her right hand into and within her left personal space, she rotated her trunk to the left (Fig. 6). In doing so, she

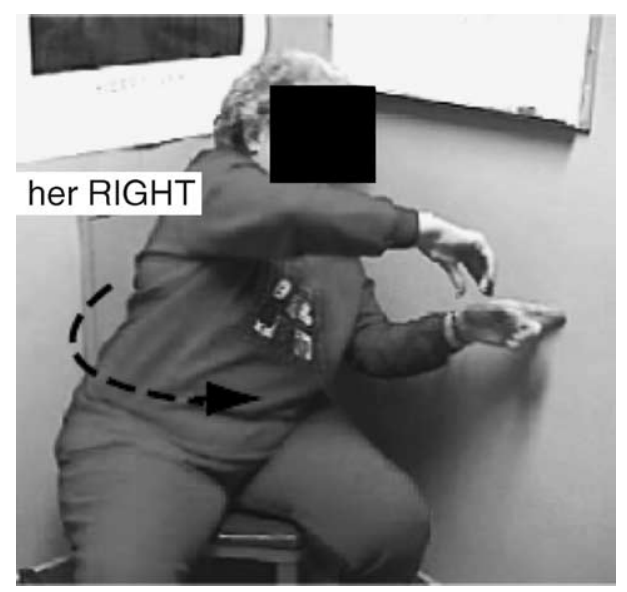

Fig. 6. Patient NG demonstrates how an object moves from the centre to the left border of the scene. In the photo she has reached the leftmost point of her demonstration. Instead of moving her right hand from the body-midline to the left border of her left personal space, she rotated her trunk in such a way that her right hand continued to move leftwards but still within her right personal space. 
extended her right personal space and was able to move her right hand further to the left within her right personal space.

\section{Discussion}

In the present study, the split-brain patients differed significantly from the two control groups in their use of space. With their right hand, they shifted the centre of the gestured scene to the right in relation to their body-midlines, and they shifted the left border of the gestured scene to the right and placed it in front of their body-midlines. With their left hand, they performed similarly to the two control groups, placing the centre of the gestured scene in front of their body-midlines and the right and left borders in the corresponding right and left personal spaces. Hence, in the split-brain patients' right-handed demonstrations, the whole scene was shifted to the right with the left border being placed in front of the body-midline and the centre being shifted into the right personal space. These results suggest a neglect of left personal space in the split-brain patients when gestures are accomplished with the right hand. No sign of hemi-spatial neglect was found with the left-handed demonstrations, the gestured scenes being, as for the two control groups, symmetrical with the body-midline.

The results are in accordance with our basic assumption as outlined in the introduction that in the split-brain patients, left hemispheric use of space is reflected by right-hand gestures while left hand gestures represent right hemisphere space use. Considering that, even under experimental conditions, the ipsilateral motor control of the right hand achieved by the right hemisphere is very limited (Trope et al., 1987; Zaidel \& Sperry, 1977), it is sensible to attribute the hemi-spatial neglect in right-hand gestures to left hemisphere (dys)function. This is further supported by the fact that if ipsilateral pathways had been used to control the right hand, no hemi-spatial neglect should have been observed during right-hand gesturing since normal use of space with the left hand (right hemisphere control) was demonstrated.

In the group with partial callosotomy, no neglect was found in LM who has spared fibres in the mid-trunk and in the rostrum of the corpus callosum. In contrast, GS, who has a 7/8 section with spared splenium and rostrum showed signs of left hemi-spatial neglect in his right-hand gestures. The localisation of the section in GS concurs with the lesion patterns found in neglect patients with spontaneously occurring callosal lesions involving left parasagittal frontoparietal areas (Sine et al., 1984), the anterior two third of the corpus callosum (Goldenberg, 1986), and the posterior half of the genu plus the whole trunk of the corpus callosum (Kashiwagi et al., 1990). Considering these results we tentatively suggest that a functional splenium (patient GS) does not prevent the occurrence of neglect while spared callosal fibres in the mid-trunk (patient LM) do. This assumption is roughly compatible with the clinico-anatomical localisation of neglect being associated with damage to posterior parietal cortex (Bisiach, 1999; Vallar \& Perani, 1986), as fibres originating from this region are known to interconnect in the caudal trunk and isthmus of the corpus callosum (Witelson, 1989). It should be pointed out, however, that Karnath, Ferber, and Himmelbach (2001) have recently reported that pure neglect occurs with damage to the right superior temporal gyrus where fibres interconnect predominantly in the isthmus of the corpus callosum (Witelson, 1989). Studies are therefore necessary to investigate further the association of spared callosal fibres with neglect. In this context, it is noteworthy that to date, an estimate of the extent of a callosal section in humans is qualitative in nature because no probability maps are available from MRI scans (Bermudez \& Zatorre, 2001) (Bermudez 2002, personal communication), and the existing map is largely based on extrapolation from the macaque's corpus callosum (Witelson, 1989). These factors may explain why the localisation of the spared fibres in LM does not perfectly overlap with the localisation of the callosal fibres that are theoretically relevant to prevent the occurrence of neglect.

In the split-brain group, NG differed from the others in that she demonstrated some ability to spatially coordinate her left hand actions with the right-hand performance. For responses in which her right hand centre placement preceded the left hand centre placement, her left hand seemed to adapt by also shifting the centre to the right. In these responses, NG's left hand also touched the right hand if it was required by the animation. In contrast, the reverse pattern, i.e. the right hand touched the left hand, was never displayed even if required by the animation. In those animations in which the right hand should have touched the left hand, there was either no touch or the left hand wrongly touched the right hand. It is plausible that NG's left hand was able to adapt to the right hand centre placement in right personal space and to touch the right hand at that location because her left hand (right hemisphere) gesture space also contained the right-hand gesture space. However, right hand confinement to right personal space did not seem to be the only reason why the right hand could not react to the left hand. The right hand was able to move to the body-midline in order to place the left border at that location but it was unable to touch the left hand in that same position. It seemed as if the right hand was unable to detect and react to the left hand even if the left hand was located in the right personal space. This is consistent with the long-standing observation that the disconnected left hemisphere ignores and denies experiences originating in the right hemisphere (Zaidel, 1978). Further, in four responses in the horizontal dimension, NG's left hand placed the centre at the same location as the right hand, i.e. shifted to the right, but it placed the centre further away from the body than the right hand. This observation concurs with several reports on neglect in the sagittal dimension (Halligan \& Marshall, 1991; Shelton, Bowers, \& Heilman, 1990; Vuilleumier, Valenza, Mayer, Reverdin, \& Landis, 1998) and suggests that in NG, the spatial frames of 
the right and left-handed demonstrations differ in both the horizontal and sagittal dimensions.

From a theoretical standpoint, the split-brain patients' right hand rightward shifts of the left border and of the midline of the scene could be interpreted as evidence for directional or hemi-spatial akinesia (Heilman et al., 1993; Meador, Watson, Bowers, \& Heilman, 1986) or as the "crossed avoiding" reaction described for callosal degeneration in Marchiafava-Bignami disease (Lechevalier, Andersson, \& Morin, 1977). However, there are several pieces of evidence that directional hypokinesia is unrelated to left hemi-spatial neglect. No directional hypokinesia for leftward movements was found in several previous studies on right-brain damaged patients with left hemi-spatial neglect (Ishiai, Watabiki, Lee, Kanouchi, \& Odajima, 1994; Mijovic, 1991; Perri, Bartolomeo, \& Gainotti, 2000). Marshall, Halligan, and Robertson (1993) have put forward another objection to the hypokinetic explanation of centre shifts in line bisection. Patients who showed a rightward shift in bisecting long horizontal lines demonstrated a leftward shift in bisecting short horizontal lines (Halligan \& Marshall, 1988; Tegnér \& Levander, 1991) such that the left hemi-spatial neglect found in long line bisection could not be secondary to directional hypokinesia. In addition, akinesia has been defined as "dysfunction of the systems necessary to activate motor neurons" (Heilman et al., 1993, p. 282). In the present study, the split-brain patients' right hand neglect of the left personal space rather reflects a "dysfunction" in the planning phase of the spatial coordinates for the right hand motor action. The spatial frame for the right-hand gestures seems to be conceptualised for the right personal space only.

The present results can be related to Karnath's model (Beschin, Cubelli, Della Sala, \& Spinazzola, 1997; Karnath, Schenkel, \& Fischer, 1991) which proposes that the trunk midline constitutes the physical anchor for calculating the internal egocentric coordinate frame representing body position with respect to external objects. In the present study, the healthy controls seemed to use their body-midlines as reference for the placement of the centre of the scene. The split-brain patients, in turn, shifted the centre of their gestural demonstration to the right when gesturing with the right hand. This finding concerning the left hemisphere in split-brain patients is compatible with the assumption of a rightward displacement of the subjective middle in patients with left hemi-spatial neglect as proposed by Karnath.

Furthermore, the observation that NG rotates her trunk to the left underlines the relevance of trunk orientation. Karnath suggests that the spatial orientation of the trunk midline divides our perception of space into an egocentric right and left, a decisive factor for determining the neglected space. By rotating her trunk to the left, NG extended her right personal space and was able to continue to move her right hand-within her right space-further to the left.

As the subjects had to demonstrate the content immediately after the animation had been shown, short-term mem- ory was involved. The low detail score in the split-brain group might reflect a deficit in attention and/or memory. However, important for the present results, it should be emphasised that details of the left hemi-space in the animation were not depicted less often than details of the right hemi-space. Therefore, details that took place in the left space of the animation must have been perceived and mentally represented just as details in the right hemi-space were. Hence, the neglect of left space in the split-brain patients' right-hand gestures cannot be explained by a perceptual and representational neglect (Bisiach, 1999). A neglect gradient (Kinsbourne, 1993) in the perception or the mental representation of the scene cannot be totally ruled out, as our methodology did not permit a quantitative assessment if the leftmost part of the animation scene was depicted in gesture. Nevertheless, even if a neglect gradient in the perception of the animation scene was present, this would not explain the total neglect of left personal space in the split-brain patients' right-handed gestures.

The split-brain patients' gestured display in their right personal spaces of events that were in the left space of the animation scene could be interpreted as allochiria in a broad sense, i.e. as a transposition from left to right (see also Halligan, Marshall, \& Wade, 1992; Heilman \& Valenstein, 1979). However, in their right-handed demonstrations, the split-brain patients did not exclusively transpose the left space of the animation scene to their right personal spaces, but they displayed the whole scene in their right personal spaces with a preservation of the original left-right orientation. Thus, there were few responses in the data set that would fit a strict definition of allochiria. In those responses, the patients gave a mirror presentation in their right personal spaces of what had happened in the left animation space. If the animation object moved from the left side of the screen to the centre and back, the patient depicted the object movement from the right side of their right personal space to the plane of the body-midline and back.

Our findings confirm McNeill and Pedelty's observation in NG (McNeill \& Pedelty, 1995) that her spontaneous right-handed gestures were confined to the right personal space. We assume that the discrepancy between the findings of signs of neglect in our study and their absence in previous neuropsychological studies is attributable to the nature of the methodologies. While our design enables the observation of spontaneous use of space in gestures, neuropsychological examinations test the ability to use space on command. The manifestation of spontaneous preference for right hemi-space by the right hand is possibly overridden if a specific use of space in motor action is required. Furthermore, in the present study, the use of space is determined by imagined targets in space, e.g. to move the hand to the left border of the imagined scene, while previous studies tested the use of space specified by actual visual targets (Plourde \& Sperry, 1984; Zaidel, 1979; Zaidel et al., 1981).

The present findings in the split-brain patients are comparable to the reports of left hemi-spatial neglect in right 
hand motor actions in patients with spontaneous callosal disconnection (Goldenberg, 1986; Kashiwagi et al., 1990; Sine et al., 1984). While callosal section can result in two independent orienting systems for visual attention (Arguin et al., 2000; Holtzman et al., 1984; Zaidel et al., in press), this does not seem to apply to the conceptualisation of spatial coordinates for motor actions. In the present experiment, the split-brain patients' right hand, which is controlled by the disconnected left hemisphere, was not able to use the whole personal space for gestural demonstration but the left hand was able to do so. Hence, the right hemisphere seems to be specialised for using the whole personal space for gestural demonstrations (and possibly other types of motor action). Therefore, we tentatively suggest that in the present experiment, the left hemisphere is dependent on the right hemisphere's spatial competence. Thus, the present results do not agree with the proposition that hemi-spatial neglect in left hemisphere functions results from suppression by the right hemisphere. Rather, it appears that neglect in left hemisphere functions can be found in the absence of right hemisphere interference via callosal pathways. Our results support the theoretical models of neglect that emphasise hemispheric asymmetry, where the left hemisphere is specialised for the right side of space while the right hemisphere processes both sides of spaces (Halligan et al., 1992; Heilman \& Valenstein, 1979; Mesulam, 1981).

\section{Acknowledgements}

This study was supported by the German Research Association grants LA 1249/1-1 and LA 1249/1-2, and by the Language and Cognition Group of the Max Planck Institute for Psycholinguistics. In particular, we wish to thank Monique Lindeman for the coding of the extensive video material, Annie Le Bire for her perpetual "no problem", and the patients and control subjects for their participation in the study.

\section{References}

Akelaitis, A. J. (1945). Studies on the corpus callosum. IV. Diagonistic dyspraxia in epileptics following partial and complete section of the corpus callosum. American Journal of Psychiatry, 101, 594-599.

Arguin, M., Lassonde, M., Quattrini, A., Del Pesce, M., Foschi, N., \& Papo, I. (2000). Divided visuo-spatial attention systems with total and anterior callosotomy. Neuropsychologia, 38, 283-291.

Berlucchi, G., Aglioti, S., \& Tassinari, G. (1997). Rightward attentional bias and left hemisphere dominance in a cue-target light detection task in a callosotomy patient. Neuropsychologia, 35, 941-952.

Bermudez, P., \& Zatorre, R. J. (2001). Sexual dimorphism in the corpus callosum: Methodological considerations in MRI morphometry. NeuroImage, 13, 1121-1130.

Beschin, N., Cubelli, R., Della Sala, S., \& Spinazzola, L. (1997). Left of what? The role of egocentric coordinates in neglect. Journal of Neurology, Neurosurgery and Psychiatry, 63, 483-489.

Bisiach E. (1999). Unilateral neglect and related disorders. In G. Denes, \& L. Pizzamiglio (Eds.), Handbook of clinical and experimental neuropsychology (pp. 479-496). Hove, UK: Psychology Press.
Bisiach, E., Pizzamiglio, L., Nico, D., \& Antonucci, G. (1996). Beyond unilateral neglect. Brain, 119, 851-857.

Bogen, J. E. (1969). The other side of the brain. I. Dysgraphia and dyscopia following cerebral commissurotomy. Bulletin of the Los Angeles Neurological Societies, 34, 73-105.

Bogen J. E. (1993). The callosal syndromes. In K. M. Heilman, \& E. Valenstein (Eds.), Clinical neuropsychology (pp. 337-408). New York: Oxford University Press.

Bogen, J. E. (2000). Split-brain basics: Relevance for the concept of one's other mind. The Journal of the American Academy of Psychoanalysis, 28, 341-369.

Bogen, J. E., Schultz, D. H., \& Vogel, P. J. (1988). Completeness of callosotomy shown by magnetic resonance imaging in the long term. Archives of Neurology, 45, 1203-1205.

Brugman, H., \& Kita, S. (1995). Impact of digital video technology on transcription: A case of spontaneous gesture transcription. Ars. Semiotica, 18, 95-112.

Campbell, A. L., Bogen, J. E., \& Smith, A. (1981). Disorganization and reorganization of cognitive and sensorimotor functions in cerebral commissurotomy. Brain, 104, 493-511.

Feinberg, T. E., Schindler, R. J., Flanagan, N. G., \& Haber, L. D. (1992) Two alien hand syndromes. Neurology, 49, 19-24.

Gazzaniga, M. S., Bogen, J. E., \& Sperry, R. W. (1967). Dyspraxia following division of the cerebral commissures. Arch. Neurol., 16, 606-612.

Geschwind, N., \& Kaplan, E. (1962). A human cerebral deconnection syndrome. Neurology, 12, 675-685.

Geschwind, D. H., Iacoboni, M., Mega, M. S., Zaidel, D. W., Cloughesy, T., \& Zaidel, E. (1995). Alien hand syndrome: Interhemispheric motor disconnection due to a lesion in the midbody of the corpus callosum. Neurology, 45, 802-808.

Goldenberg, G. (1986). Neglect in a patient with partial callosal disconnection. Neuropsychologia, 24, 397-403.

Halligan, P. W., \& Marshall, J. C. (1988). How long is a piece of string? A study of line bisection in a case of visual neglect. Cortex, 24, 321-328.

Halligan, P. W., \& Marshall, J. C. (1991). Left neglect for near but not far space in man. Nature, 350, 498-500.

Halligan, P. W., Marshall, J. C., \& Wade, D. T. (1992). Left on the right: Allochiria in a case of left visuo-spatial neglect. Journal of Neurology, Neurosurgery and Psychiatry, 55, 717-719.

Heilman, K. M., \& Valenstein, E. (1979). Mechanisam underlying hemispatial neglect. Annals of Neurology, 5, 166-170.

Heilman K. M., Watson R. T., \& Valenstein E. (1993). Neglect and related disorders. In K. M. Heilman, \& E. Valenstein (Eds.), Clinical neuropsychology (3rd ed., pp. 279-336). New York: Oxford University Press.

Holtzman J. D., Volpe B. T., \& Gazzaniga M. S. (1984). Spatial orientation following commissural section. In R. Parasuram, \& D. R. Davies (Eds.), Varieties of attention (pp. 375-394). Orlando: Academic Press.

Ishiai, S., Watabiki, S., Lee, E., Kanouchi, T., \& Odajima, N. (1994) Preserved leftward movement in left unilateral spatial neglect due to frontal lesions. Journal of Neurology, Neurosurgery and Psychiatry, $57,1085-1090$

Joynt, R. J. (1977). Inattention syndromes in split-brain man. Advances in Neurology, 18, 33-39.

Karnath, H. O., \& Niemeier, M. (2002). Task-dependent differences in the exploratory behaviour of patients with spatial neglect. Neuropsychologia, 40, 1577-1585.

Karnath, H. O., Schenkel, P., \& Fischer, B. (1991). Trunk orientation as the determining factor of the 'contralateral' deficit in the neglect syndrome and as the physical anchor of the internal representation of body orientation in space. Brain, 114, 1997-2014.

Karnath, H. O., Ferber, S., \& Himmelbach, M. (2001). Spatial awareness is a function of the temporal not the posterior parietal lobe. Nature, 411, 950-953.

Kashiwagi, A., Kashiwagi, T., Nishikawa, T., Tanabe, H., \& Okuda, J. (1990). Hemispatial neglect in a patient with callosal infarction. Brain, 113, 1005-1023. 
Kinsbourne M. (1993). Orientational bias model of unilateral neglect: Evidence from attentional gradients within hemispace. In I. $\mathrm{H}$. Robertson, \& J. C. Marshall (Eds.), Unilateral neglect: Clinical and experimental studies (pp. 63-86). Hove, UK: Lawrence Erlbaum.

Làdavas, E., Del Pesce, M., Mangum, G. R., \& Gazzaniga, M. S. (1994). Variations in attentional bais of the disconnected cerebral hemispheres. Cognitive Neuropsychology, 11, 57-74.

Lausberg, H., Göttert, R., Münßinger, U., Boegner, F., \& Marx, P. (1999). Callosal disconnection syndrome in a left-handed patient due to infarction of the total length of the corpus callosum. Neuropsychologia, $37,253-265$.

Lausberg, H., Cruz, R. F., Kita, S., Zaidel, E., \& Ptito, A. (2003). Pantomime to visual presentation of objects: Left hand dyspraxia in patients with complete callosotomy. Brain, 126, 343-360.

Lechevalier, B., Andersson, J. C., \& Morin, P. (1977). Hemispheric disconnection syndrome with a crossed avoiding reaction in a case of Marchiafava-Bignami disease. Journal of Neurology, Neurosurgery and Psychiatry, 40, 483-497.

Marshall J. C., Halligan P. W., \& Robertson I. H. (1993). Contemporary theories of unilateral neglect: A critical review. In I. H. Robertson, \& J. C. Marshall (Eds.), Unilateral neglect: Clinical and experimental studies (pp. 311-329). Hove, UK: Lawrence Erlbaum.

McNeill D., \& Pedelty L. L. (1995). Right brain and gesture. In K. Emmorey, \& Reilty (Eds.), Language, gesture, and space (pp. 63-85). Hove, UK: Lawrence Erlbaum.

Meador, K. J., Watson, R. T., Bowers, D., \& Heilman, K. M. (1986). Hypometria with hemispatial and limb motor neglect. Brain, 109, 293305.

Mesulam, M. M. (1981). A cortical network for directed attention and unilateral neglect. Annals of Neurology, 10, 309-325.

Mijovic, D. (1991). Mechanisms of visual spatial neglect. Absence of directional hypokinesia in spatial exploration. Brain, 114, 15751593.

Milner, B., \& Taylor, L. (1972). Right-hemisphere superiority in tactile pattern-recognition after cerebral commissurotomy: Evidence for non-verbal memory. Neuropsychologia, 10, 1-15.

Perri, R., Bartolomeo, P., \& Gainotti, G. (2000). Lack of impairments on leftward and rightward line extension tasks in neglect patients. International Journal of Neuroscience, 103, 101-113.

Plourde, G., \& Sperry, R. W. (1984). Left hemisphere involvement in left spatial neglect from right-sided lesions. Brain, 107, 95-106.

Pollmann, S., \& Zaidel, E. (1998). The role of the corpus callosum in visual orienting: Importance of interhemispheric visual transfer. Neuropsychologia, 36, 763-774.

Robertson, I. H., \& North, N. T. (1992). Spatio-motor cuing in unilateral neglect: The role of hemispace. Neurospychologia, 30, 553-563.

Robertson, I. H., \& North, N. T. (1994). One hand is better than two: Motor extinction of left hand advantage in unilateral neglect. Neuropsychologia, 32, 1-11.
Shelton, P. A., Bowers, D., \& Heilman, K. M. (1990). Peripersonal and vertical neglect. Brain, 113, 191-205.

Sine, R. D., Soufi, A., \& Mahendra, S. (1984). Callosal syndrome: Implications for understanding the neuropsychology of stroke. Archives of Physical Medicine and Rehabilitation, 65, 606-610.

Tanaka, Y., Yoshida, A., Kawahata, N., Hashimoto, R., \& Obayashi, T. (1996). Diagonistic dyspraxia—clinical characteristics. Brain, 119, $859-873$.

Taylor, L. (1970). Somethetic threshold after commisural section in man. Neurology, 20, 378

Tegnér, R., \& Levander, M. (1991). The influence of stimulus properties on visual neglect. Journal of Neurology, Neurosurgery and Psychiatry, 54, 881-887.

Trope, I., Fishman, B., Gur, R. C., Sussman, N. M., \& Gur, R. E. (1987). Contralateral and ipsialteral control of fingers following callosotomy. Neuropsychologia, 25, 287-291.

Vallar, G., \& Perani, D. (1986). The anatomy of unilateral neglect after right hemisphere stroke lesions. A clinical/CT-scan correlation study in man. Neuropsychologia, 24, 609-622.

Volpe B.T. (1985). Observations of motor control in patients with partial and complete callosal section. In A. G. Reeves (Ed.), Epilepsy and the corpus callosum (pp. 381-391). New York: Plenum Press.

Volpe, B. T., Sidtis, J. J., Holtzman, J. D., Wilson, D. H., \& Gazzaniga, M. S. (1982). Cortical mechanisms involved in praxis: Observations following partial and complete section of the corpus callosum in man. Neurology, 32, 645-650.

Vuilleumier, P., Valenza, N., Mayer, E., Reverdin, A., \& Landis, T. (1998). Near and far visual space in unilateral neglect. Annals of Neurology, 43, 406-410.

Witelson, S. F. (1989). Hand and sex differences in the isthmus and genu of the human corpus callosum. A postmortem morphological study. Brain, 112, 799-835.

Zaidel E. (1978). Concepts of cerebral dominance in the split brain. In P. A. Buser, \& A. Rougeul-Buser (Eds.), Cerebral correlates of conscious experience (INSERM Symposium No. 6, pp. 263-284). Amsterdam: Elsevier.

Zaidel, E. (1979). Performance on the ITPA following cerebral commissurotomy and hemispherectomy. Neuropsychologia, 17, 259280.

Zaidel, E. (1998). Stereognosis in the chronic split brain: Hemispheric differences. Neuropsychologia, 36, 1033-1047.

Zaidel, D., \& Sperry, R. W. (1977). Some long-term motor effects of cerebral commissurotomy in man. Neuropsychologia, 15, 193-204.

Zaidel, E., Zaidel, D. W., \& Sperry, R. W. (1981). Left and right intelligence: Case studies of Ravens' progressive matrices following brain bisection and hemidecortication. Cortex, 17, 167-186.

Zaidel E., Iacoboni M., Zaidel D.W., \& Bogen J.E. (in press). The callosal syndromes. In K. M. Heilman, \& E. Valenstein (Eds.), Clinical neuropsychology (4th ed.). New York: Oxford University Press. 